\title{
Management of Patients with Malignancy before Heart Transplantation
}

\author{
Wilhelm Mistiaen ${ }^{1,2, *}$ \\ ${ }^{1}$ Faculty of Medicine \& Health Sciences, University of Antwerp, Universiteitsplein 1, 2610, Antwerp, Belgium \\ ${ }^{2}$ Dept. Health \& Well-being, Artesis-Plantijn University of Applied sciences, 2000 Antwerp, Belgium
}

\begin{abstract}
Background: Heart transplantation and malignancy connect in several ways. Cancer can be an incidental finding, cancer treatment can also be the cause of end-stage heart failure, for which heart transplantation is necessary or malignancy can occur after transplantation, because of immune suppression.

Methods: This is a database search on Web of Science from 2010 on, with the term "heart transplant* AND (cancer OR malignancy)". This resulted in 3767 titles.

Results: Eight articles contained sufficient information about survival. In most series, a history of pre-transplant malignancy (PTM) did not affect survival, except for hematologic malignancy or short cancer-free pre-transplant interval. Other patient-related factors were usually more important. The effect of PTM on the rate of post-transplant malignancy followed a comparable pattern. In patients with chemotherapy-related cardiomyopathy, other causes than malignancy were more important.

Conclusions: Outcome for heart transplantation in patients with PTM is acceptable in terms of survival, and occurrence post-transplant malignancy. An appropriate PTM-free interval of two to five year seems necessary. Hematologic PTM have a worse outcome. Although the limitations of this review warrant caution with the interpretation of its results, increased post-transplant screening for malignancy and of use of proliferation signal inhibitors, due to their antineoplastic activity, could be cornerstones of the management of these patients.
\end{abstract}

Keywords: Pre-transplant malignancy, Post-transplant malignancy, Orthotopic heart transplant, Survival.

\section{INTRODUCTION}

Malignancy and orthotopic heart transplantation (OHT) have several connections. First, many patients, needing $\mathrm{OHT}$ have shared risk factors for heart disease but also for cancer. This is due to a certain lifestyle. The incidence of pre-transplant malignancy (PTM) can be 3\% [1] or more [2]. Second, treatment of cancer by chemotherapy such as anthracyclines [3-7] cause damage of the cardiomyocytes and can result in chemotherapy-related cardiomyopathy (CCMP). This is especially true for pediatric patients, in whom this type of treatment is used [8]. In spite of preventive measures, end-stage heart failure (ESHR) might develop in 2 to $4 \%$ of these patients [3], for whom OHT is the only option to alter its natural course [9]. Since the number of cancer survivors increases, the number and the relative rate of candidates for $\mathrm{OHT}$ with such history is also on the rise, and this can be over $5 \%$ [10]. This is partly because improved detection [3, 5, 9]. Serious comorbid conditions such as a PTM could limit post-transplant survival or prohibit an adequate immune suppression. For this reason, PTM was a

"Address correspondence to this author at the Faculty of Medicine \& Health Sciences, University of Antwerp, Universiteitsplein 1, 2610, Antwerp, Belgium; Tel: 3238305002 ;

E-mail: wilhelm.mistiaen@uantwerpen.be; wilhelm.mistiaen@ap.be relative contraindication to transplantation for a long time [10, 11]. This view is shifting and this has its importance for young adult and pediatric patients considered for OHT who would have otherwise a long life expectancy because of improved cancer treatment $[3-5,8]$. Third, post-transplant malignancy is a consequence of immune suppression and remains a major cause of post-OHT, mortality [10, 12-14], especially with increasing duration of follow-up [15, 16], without reaching a plateau [16]. The incidence of malignancy after $\mathrm{OHT}$ is high $[11,12,14,17]$, compared to other organs transplants. In European series, this could reach $30 \%$ at about 9 year follow-up and could be responsible for more than $20 \%$ of the fatalities after OHT [12]. The type and the dose of immune suppressing regimens play an important role herein [15]. The scope of this review deals mainly with the effect of pre-transplant malignancy on post-OHT outcome. The following research questions are

- what is the effect of PTM and of CCMP on survival after OHT

- what is their effect on the rate of post-transplant malignancy

The outcome could alter the management of this difficult patient group. 


\section{MATERIALS AND METHODS}

This was a database search on Web of Science from 2010 on, with the term "heart transplant* AND (cancer OR malignancy)". This rather simple MeSH term allowed a search for a broad scope of articles. Laboratory experiments, animal experiments, transplantation other than $\mathrm{OHT}$, organ transplant in general (unless results of OHT were investigated separately), cases, reviews, meeting abstracts, primary cardiac tumors, outcome not being mortality/posttransplant malignancy, skin cancer as sole outcome were excluded, as well as reports about radiation heart disease since this is another condition with a different outcome. This resulted in 3767 titles. The first round of selection was on title and on abstract dealing with clinical series dealing with $\mathrm{OHT}$ and including pretransplant malignancy. Ninety-three articles were eligible for full-text screening.

\section{RESULTS}

Eight articles were included, which dealt with the effect of pre-transplant malignancy on outcome after $\mathrm{OHT}$ (shown in Table 1). All studies were observational and of retrospective nature. These series compared patients with PTM versus patients without PTM, except for one, in which different intervals between PTM and OHT were studied [1]. In one series, the effect of hematologic PTM was investigated separately [10].In almost all series, the patient group with PTM was much smaller, compared to those without PTM. This indicated that PTM is relatively rare. Except for one series [1], all studies were derived from large databases (such as United Network for Organ Sharing or UNOS), often linked with cancer databases. Two series were paediatric $[4,8]$ and three series compared OHT for CCMP versus OHT for other reasons [3, 5, 8].

Table 1: Survival after Heart Transplantation

\begin{tabular}{|c|c|c|c|c|c|c|c|c|c|c|}
\hline Author (ref) & Group & Age & $\mathbf{N}^{\circ}$ & Incl period & 1y-surv & $2 y$-surv & 3y-surv & 5y-surv & $10 y$-surv & $\mathrm{p}$-value \\
\hline \multirow[t]{2}{*}{ Fernandez 2010 (9) } & no PTM & 53 & 583 & $1991-2009$ & $82 \%$ & & $76 \%$ & $70 \%$ & & \\
\hline & PTM & 53 & 12 & IBID & $75 \%$ & & $75 \%$ & $56 \%$ & & 0.70 \\
\hline \multirow[t]{3}{*}{ Sigurdardottir 2012 (1) } & PTM >5y & $<40$ & 24 & $1983-2011$ & $100 \% *$ & $90 \% *$ & $73 \% *$ & $72 \% *$ & $58 \% *$ & \\
\hline & PTM 1-5y & $<40$ & 18 & IBID & $82 \%{ }^{*}$ & $80 \%{ }^{*}$ & $78 \% *$ & $78 \% *$ & $38 \% *$ & 0.93 \\
\hline & PTM $<1 y$ & $<40$ & 71 & IBID & $80 \%{ }^{*}$ & $68 \% *$ & $58 \% *$ & $55 \% *$ & $32 \% *$ & 0.043 \\
\hline \multirow[t]{3}{*}{ Beaty $2013(10)$} & no PTM & 55 & 18586 & $2000-2011$ & $88 \%{ }^{*}$ & $84 \% *$ & $78 \%{ }^{*}$ & $73 \% *$ & & \\
\hline & other PTM & 55 & 961 & IBID & $88 \%{ }^{*}$ & $80 \%{ }^{*}$ & $76 \% *$ & $72 \% *$ & & NS \\
\hline & hem PTM & 55 & 146 & IBID & $78 \%{ }^{*}$ & $72 \%{ }^{*}$ & $72 \%$ * & $69 \% *$ & & $<0.01$ \\
\hline \multirow[t]{2}{*}{ Shah 2013 (4) } & no PTM & 7 & 7062 & $1987-2011$ & $84,4 \%$ & & & $73,8 \%$ & $57,7 \%$ & \\
\hline & PTM & 12 & 107 & IBID & $90,6 \%$ & & & $80,3 \%$ & $65 \%$ & 0.18 \\
\hline \multirow[t]{2}{*}{ Delgado 2016 (11) } & no PTM & 54 & 4484 & $1984-2010$ & $93 \%$ & & & $79 \%$ & $65 \%$ & \\
\hline & PTM & about 60 & 77 & IBID & $95 \%$ & & & $74 \%$ & $51 \%$ & 0.048 \\
\hline \multirow[t]{2}{*}{ Bock 2017 (8) } & no CCMP & 12 & 136 & $1993-2014$ & $92 \%$ & & & $80 \%$ & & \\
\hline & CCMP & 12 & 62 & IBID & $92 \%$ & & & $74 \%$ & & 0.37 \\
\hline \multirow[t]{2}{*}{ Oliveira 2012 (3) } & no CCMP & 49 & 8890 & $2000-2008$ & $87 \%$ & & $81 \%$ & $74 \%$ & & \\
\hline & CCMP & 46 & 232 & IBID & $86 \%$ & & $79 \%$ & $71 \%$ & & 0.19 \\
\hline Lenneman 2013 (5) & no CCMP & 44 & 51312 & $1987-2011$ & $87 \%{ }^{*}$ & $85 \% *$ & $82 \% *$ & $75 \% *$ & $58 \% *$ & \\
\hline (adjusted) & CCMP & 44 & 453 & IBID & $91 \% *$ & $88 \%{ }^{*}$ & $85 \% *$ & $83 \% *$ & $67 \%{ }^{*}$ & 0.026 \\
\hline \multirow[t]{2}{*}{ "(not adjusted)" } & no CCMP & 52 & 51312 & IBID & $85 \%{ }^{*}$ & $83 \% *$ & $78 \%{ }^{*}$ & $72 \%{ }^{*}$ & $54 \% *$ & \\
\hline & CCMP & 52 & 453 & IBID & $88 \%$ * & $84 \% *$ & $80 \%$ * & $74 \%$ * & $57 \%$ * & 0.198 \\
\hline
\end{tabular}

Legend: CCMP: chemotherapy-related cardiomyopathy; hem: hematologic; incl.: inclusion; $\mathrm{N}^{\circ}$ : number; NS: not significant; PTM: pre-transplant malignancy; surv: survival; y: year; percentages indicated with * are estimations, derived from survival curves, percentages without asterisk, are derived from tables in the referred articles. 


\subsection{Mortality}

Some articles did not give survival rates as a percentage but offered curves for freedom of mortality (the non-parametric Kaplan-Meier curve), which indicated these percentages with some accuracy $[1,5$, 10]. In these series, the $p$-value is important to demonstrate the effect of PTM on outcome after OHT. The three articles dealing specifically with OHT for CCMP showed that post-transplant survival was not lower in patients who received OHT for CCMP. Reasons for this observation were young age, lower BMI and fewer comorbid conditions [3, 5, 8]. Two series were of pediatric nature $[4,8]$. Another series dealt with the effect of the preoperative interval between malignancy and OHT for any ESHF on posttransplant survival, but made no comparison with patients without a history of PTM. This series showed that with shorter interval, postoperative survival decreased significantly [1]. Overall, six of the eight articles could not demonstrate a significant difference in post-transplant survival between patients with and without a PTM [3-5, 8-10]. However, the series investigating the effect of hematologic PTM on posttransplant outcome separately showed that this type of PTM resulted in a significantly worse post-transplant survival and this effect was larger than any demographic or comorbid factor, except for new onset dialysis [10]. Only in one series, patients with PTM had a decreased survival, but this became only evident after 5 years, and only in a univariate analysis [11].

In five manuscripts $[3,5,8,10,11]$, a multiple regression analysis was performed. This showed the predictors for survival after OHT. The predictors for 5year mortality after $\mathrm{OHT}$ were new onset dialysis (as most important, with a hazard ratio of 4.18). Of the different types PTM, only hematologic malignancies were predictive (with a hazard ratio of 1.65 and $p<0.01)$. Other demographic and comorbid conditions were less important or not significant [10]. Another series could not identify PTM as predictor, even not after adjustment for gender and era, although male gender and era before 1993 were predictive [11]. In a pediatric series of patients with CCMP, listing in earlier era, need for a ventricular assist device and female gender were predictors for mortality after $\mathrm{OHT}$, but CCMP was not [8]. A second series exploring the effect of CCMP on outcome confirmed this observation at 1 and at 5 years after OHT [3]. Remarkably, adjustment for age, gender and a history for PTM by the Cox proportional hazard regression model showed better post-OHT results for the CCMP group. A few patients with PTM, however, were also part of the non-CCMP group. Obviously, their cancer treatment has not lead to the development of CCMP [5]. Overall, four out of five series showed that post-transplant survival of patients with a history of PTM or CCMP had not decreased. Two remarkable designs dealt with prior malignancy, present in cadaveric donors $[18,19]$. Although presence of such malignancy was a predictor for 10-year survival in the first series, this effect disappeared after propensity score matching or after conditioning upon 1-year post-transplant survival. Factors unrelated to malignancy were of more importance [18]. In the other series, a history of hematologic and otorhinolaryngologic malignancy in donors seemed more a problem for $\mathrm{OHT}$ in terms of graft and patient survival. This was not the case for other donor malignancies [19]. These series indicate that with careful risk analysis for every donor, the organ pool for transplant expansion is possible.

\subsection{Post-Transplant Neoplasms}

Only in three series, the incidence of post-transplant malignancy and the survival were presented [1, 4, 12]. The series comparing the effect of three different intervals between malignancy and OHT showed that with an interval of 5 year or more, incidence of posttransplant malignancy is also low if compared, to smaller intervals. However, the curve of survival was steeper that of freedom of post-transplant malignancy in the 5-year interval, while in the smaller intervals, survival and freedom of post-transplant malignancy were more resembling. This indicates that death due to post-transplant malignancy is not dominant in the group with an interval of $>5$ years, while it plays a larger role in the group with smaller intervals, which can be expected [1]. In one of the pediatric series, incidences of post-transplant malignancy remained low, and survival after one and 5 year was high. This was the only series in which rate of post-transplant malignancy and survival were studied at the same time [4]. Another series showed a gradual increase of post-transplant malignancy for patients with and without PTM, but without significant differences. Data about survival are lacking [12]. Of the three series studying the effect of $\operatorname{CCMP}[3,5,8]$ only one showed a higher rate of posttransplant malignancy and infections, but a comparable survival rate [3]. Both other series showed comparable and low rates of post-transplant malignancy $[5,8]$.

In one pediatric series, the rate of post-transplant malignancy was higher $(13 \%$, with $5.6 \%$ de novo malignancy and $5.6 \%$ post-transplant lymphoproliferative 
disease or PTLD) in the group with PTM, compared to the group without PTM $(5.4 \%, p=0.001)$. In patients with PTM, post-transplant malignancy occurred earlier, but the survival was similar [4]. In another pediatric series, infection as cause of death was $30 \%$ in the CCMP, while no deaths were due to malignancy [8]. Two adult series studied the effect of CCMP (with mainly hematologic malignancies and breast cancer as PTM) on outcome. In one series, post-transplant malignancy rate was higher in the PTM group $(5 \% \mathrm{v}$. $2 \%$, with $p=0.006$ ). This was also true for infection $(22 \%$ v. $14 \%, p=0.004)$, but neither affected survival at $1 \mathrm{y}$ or at 5y [3]. The other series offered no data for post-transplant malignancy, only the observation that death because of malignancy was comparable for both groups and of less importance compared to other causes [5]. In one series the incidence of posttransplant malignancy was 1.8 times greater $(p<0.00[)$, and this was 2.3 times higher in patients with hematologic PTM [11]. The effect of the hematologic nature of PTM in the UNOS based study is in line with this observation [10]. Hematologic malignancies have a genomic instability with an increased tendency to accumulate mutations with each mitosis [11]. In the exploration for the effect the pre-transplant cancer-free interval, the freedom from post-transplant recurrence for each group showed a parallelism with posttransplant survival: with shorter pre-transplant cancerfree interval, the recurrence of malignancy increased drastically ( $63 \%$ for the group with interval $<1$ y; $23 \%$ for the group of 1 to $5 \mathrm{y}$ and $6 \%$ for the group with interval $>5 y$ ). Mortality because of post-transplant malignancy also increased drastically with shorter cancer-free pretransplant intervals [1].). One series showed that hazard ratio of PTM on post-transplant malignancy was 1.5 , but this was not significant [16]. Another series showed a significant difference in this respect (1/96 v. $1 / 14$ ) but the absolute numbers were very low and other factors such as the type of induction treatment should be accounted for [14]. Interestingly, one series showed that skin cancer was by far the most common post-transplant malignancy, followed by lung cancer, prostate cancer and PTLD. Overall, PTM increased the risk for overall post-transplant malignancy. Posttransplant skin malignancy was significantly higher with a history pre-transplant skin malignancy (2.79 times) and pre-transplant solid malignancy (1.55 times). Solid PTM, however, had no effect on the incidence of posttransplant solid malignancy. There were no data for mortality [12]. Overall, the effect of PTM on posttransplant malignancy is mixed. However, short cancerfree pre-transplant period and hematologic nature of
PTM indicate an increase in post-transplant malignancy.

\subsection{Causes of Death}

In smaller to medium sized series, there were no recurrences of PTM. None of the post-transplant fatalities was due to PTM [8, 9]. In two CCMP groups, graft failure, acute rejection and infection were much more important for mortality than post-transplant malignancy $[3,8]$. In the third CCMP group, cardiovascular causes of post-transplant mortality were the largest for those with and without CCMP. Death from malignancy was the same for both groups [5]. A larger series showed that causes of death in the PTM patients was $32.1 \%$. For patients without PTM, this was lower $(21.3 \%)$ but not significantly different [11]. However, in one series, the pre-transplant cancer-free interval played a role: there were 50/111 deaths with a cancer-free pre-transplant interval of less than a year. Death because of malignancy was $14 / 24$ (or $58 \%$, with recurrence rate of malignancy of $63 \%$ ), for the interval between 1 and 5 year, this was $3 / 18(17 \%$, with a recurrence rate of $26 \%$ ) and for the interval of over 5 year, this was $3 / 69(4 \%$, with a recurrence rate of $6 \%)$. These figures clearly indicate the importance of this interval [1]. One pediatric series compared the group with and without PTM for the effect of post-transplant malignancy on survival. This survival was similar [4]. The type of post-transplant malignancy had an effect on mortality: for cutaneous cancer, mortality was $9 \%$ with a median survival of 13.1 years; for non-cutaneous malignancy, mortality was $76 \%$ with a median survival of 2.9 , which was significantly lower [16].

\section{DISCUSSION}

\section{Mortality}

The majority of the included manuscripts showed that the presence of PTM did not affect post-transplant survival in a significant way [3-5, 8, 9]. One series showed that post-transplant survival was only lower if these were hematologic in nature and only after 1 year, but not at 5 years. The presence of other types of PTM had no such effect. The authors separated lung transplant patients clearly from $\mathrm{OHT}$ patients, which made the study of the latter more adequate [10]. One series showed a significantly worse survival after OHT in patients with PTM but only after 5 years [1]. One series compared cancer free intervals before OHT for their effect on survival. Patients were without PTM were not included. Nevertheless, patients with a 
cancer-free interval of more than 5 years had a posttransplant survival comparable to the survival of patients without PTM from the other series. Two thirds of the patients received $\mathrm{OHT}$, the remaining patients received lung transplantation but both groups were analyzed together [1]. From these data, it is clear that hematologic PTM and PTM with short cancer-free interval result in worse outcome after OHT. The effect of the cancer-free pre-transplant interval is in line with the observations made for conventional cardiac surgery, such as CABG [20]. The multivariate analysis confirmed that the effect of PTM on post-transplant survival was largely not significant: in one series, hematologic PTM was the sole malignancy, which had a predictive effect [10]. Other factors such as need for mechanical circulatory support, comorbid conditions, gender, and transplant era were significant.

\section{Recurrences of Neoplasms and Causes of Death}

Post-transplant malignancy was higher in the PTM group in most series [4, 12], especially with short intervals [1] and hematologic PTM [11] except in one adult CCMP series [3]. The effect of the hematologic nature on this outcome could be replicated [1]. In the series focusing on the post-transplant malignancy, PTM had no significant effect, although the hazard ratio was just over 1.5 [16]. Some series had very low numbers $[1,9,14]$. Therefore, the results of small series warrant caution. Nevertheless, most series show a comparable survival after occurrence of posttransplant malignancy in both groups with and without PTM [3-5, 8, 9], but not with short interval [1] or with non-skin malignancy [16]. What is the safe interval to perform $\mathrm{OHT}$ in patients with a previous malignancy? One smaller series seems to indicate 5 years [1], which is also the case in more commonly performed cardiac surgery [20]. This is in line with the ISHLT listing criteria [21]. The type of tumor plays an important role. One should also be aware of incidentally or unexpectantly found cancers.

\section{Immune Suppressing Regimen as Confounder}

Only a few series analyzed the effect of immune suppression [11]. Continuous improvement of immune suppressing regimens during the long patient inclusion times could confound the effect of PTM on the posttransplant outcome. An example are the proliferation signal inhibitors (or inhibitors of mammalian target of rapamycin - mTOR), which were recently introduced and have antineoplastic properties [1], thereby reducing the risk for cancer after $\mathrm{OHT}$. Induction treatment, such as anti-CD3 monoclonal antibodiesOKT3 / anti-thymocyteglobulin, as preventive measure against early rejection also increased the risk for post-transplant malignancy, but this is not a universal observation [17]. Induction with Interleukin-2receptor blocking monoclonal antibodies had no such effect $[17,11]$. One series shows an increase in use of mofetilmycophenolate, tacrolimus, induction with daclizumab, antiviral prophylaxis and a decrease in use of cyclosporine, azathioprine, induction with OKT3 in the periods 1991-2000 v. 2001-2010. At the same time, they observed a decrease in post-transplant hematologic malignancies [15]. Replacing azathioprine by mofetilmycophenolate [16] and calcineurin inhibitors (cyclosporine and tacrolimus) by mTOR inhibitors as maintenance immune suppression reduced the rate of post-transplant malignancies [22]. Use of these proliferation signal (or mTOR) inhibitors such as sirolimus and everolimus have their main advantage as preventive measure, and less as rescue approach [23].

\section{Management Options for Patients with PTM}

These results indicate that $\mathrm{OHT}$ is an appropriate treatment for end-stage heart failure, even in presence of PTM. If the interval is short or if the PTM is hematologic in nature, survival after PTM is worse. However, OHT in patients with CCMP shows excellent results. This is probably due to favorable demographic profile and low comorbidity. Because of an increased rate of post-transplant malignancy in some series, a regular and thorough check-up is warranted in these patients. The newer proliferation signal inhibitors, which show an antineoplastic behavior should be used at the start of the immune suppressing regimens. This seems superior compared to a rescue approach, once a posttransplant malignancy has developed.

\section{LIMITATIONS}

All series were retrospective with inherent risks for bias. The papers included in the table were inhomogeneous. This inhomogeneity included following items.

Designs differ because of different pre-transplant factors were not uniformly included (such as need for mechanical circulatory support, need for inotrope medication, time on transplantation list, race, comorbid conditions, serology of oncogene viruses)

Multivariate analysis was not used in all series 
Large databases do not always provide clinically relevant information about PTM, such as cancer treatment modalities and cancer-free interval

- Large databases cannot make the distinction between recurrent and "de novo" malignancy

Some series have small numbers of patients with PTM, and should be interpreted cautiously

The time of patient inclusion varied between 8 and 28 years. With the improving immune suppressing regimens, this makes comparison between series, but also within series more difficult.

Two series were single center and six were based on registries and databases with risk for incomplete registration and coding errors

Two series were pediatric and six were adult (excluding patients under 17 of 18 years)

Five series investigated the effect of PTM on outcome without distinction of CCMP, although authors sometimes presented the indications for $\mathrm{OHT}$. Dilated cardiomyopathy was sometimes presented as one group. Three series studied the effect of CCMP (which are by definition patients with a history of PTM) on outcome, but with a small number of PTM in the non CCMP group

- $\quad$ Three authors offered survival curves, without specific percentages. These percentages had to be estimated. However, the p-values strengthened the message.

Authors did not always distinguish relapses of PTM and occurrence of "de novo" malignancies.

Post-transplant malignancy rates and posttransplant survival were rarely studied in on and the same series.

Except for some remarks of hematologic malignancies and skin cancer, no detailed analysis was performed for different types of malignancy in most series.

\section{CONCLUSIONS}

Although there are some discrepancies between in results of the included articles, outcome for OHT in patients with PTM is acceptable in terms of survival, relapse of PTM and "de novo" malignancy. However, post-transplant malignancy has some effect on survival after OHT. An appropriate PTM-free interval of two to five year seems desirable, but this cannot always be achieved. Hematologic PTM have a worse outcome. Post-transplant skin cancers have little impact on survival. With the introduction of proliferation signal inhibitors, results have improved, due to their antineoplastic activity.

\section{ACKNOWLEDGMENTS}

The author states that there is no acknowledgement to make

\section{ADDITIONAL MATERIALS (IF ANY)}

There are no additional materials

\section{AUTHOR CONTRIBUTIONS}

Not applicable.

\section{FUNDING}

The author declares that there has been no funding.

\section{COMPETING INTERESTS}

The author declares that no competing interests exist.

\section{LIST OF ABBREVIATIONS}

CCMP: chemotherapy related cardiomyopathy

OHT: orthotopic heart transplantation

PTLD: post-transplant lymphoproliferative disease

PTM: pre-transplant malignancy

UNOS: United Network for Organ Sharing

mTOR: mammalian target of rapamycin

OKT3: anti CD3 monoclonal antibody

\section{REFERENCES}

[1] Sigurdardottir V, Bjortuft O, Eiskjaer H, Ekmehag B, Gude E Gustafsson $\mathrm{F}$, et al. Long-term follow-up of lung and heart transplant recipients with pre-transplant malignancies. J Heart and Lung Transpl 2012; 31: 1276-1280. https://doi.org/10.1016/j.healun.2012.09.007

[2] Jäämaa-Holmberg S, Salmela B, Lemström K, Pukkala E, Lommi J. Cancer incidence and mortality after heart transplantation - A population-based national cohort study. ActaOncologica 2019; 58: 859-863. https://doi.org/10.1080/0284186X.2019.1580385 
[3] Oliveira GH, Hardaway BW, Kucheryavaya AY, Stehlik J, Edwards LB, Taylor DO. Characteristics and survival of patients with chemotherapy-induced cardiomyopathy undergoing heart transplantation. J Heart Lung Transplant 2012; 31: 805-810. https://doi.org/10.1016/j.healun.2012.03.018

[4] Shah N, Aggarwal S, L'Ecuyer T. Outcome of heart transplantation in pediatric cancer survivors. Pediatr Transplantation 2013; 17: 423-428. https://doi.org/10.1111/petr.12072

[5] Lenneman AJ, Wang L, Wigger M, Frangoul H, Herrell FE, Silverstein C, et al. Heart Transplant Survival Outcomes for Adriamycin-Dilated Cardiomyopathy. Am J Cardiol 2013; 111: 609-612. https://doi.org/10.1016/j.amjcard.2012.10.048

[6] Mistiaen W. Heart transplantation in patients with a previous malignancy: an overview. ActaCardiol 2015; 70: 123-130. https://doi.org/10.1080/AC.70.2.3073502

[7] Mistiaen W. Effect of Doxorubicin on Cardiac Myocytes: Role of Apoptosis, Autophagy and other Proteolytic Pathways. International Journal of Cardiology and Lipidology Research, 2016, 3, 31-43.

https://doi.org/10.15379/2410-2822.2016.03.02.01

[8] Bock MJ, Pahl E, Rusconi PG, Boyle GJ, Parent JJ, Twist $\mathrm{CJ}$, et al. Cancer recurrence and mortality after pediatric heart transplantation for anthracycline cardiomyopathy: A report from the Pediatric Heart Transplant Study (PHTS) group. Pediatric Transplantation.2017; 21: e12923. https://doi.org/10.1111/petr.12923

[9] Fernandez-Vivancos C, Paniagua-Martin MJ, Marzoa-Rivas R, Barge-Caballero E, Grile-Cancela Z, Recio-Mayoral A, et al. Long-Term Outcome in Heart Transplant Patients With Pretransplant Malignancies. Transplant Proc 2010; 42: 20003010.

https://doi.org/10.1016/j.transproceed.2010.08.012

[10] Beaty CA, George TJ, Kilic A, Conte JV, Shah AS. PreTransplant Malignancy: An Analysis of Outcomes after Thoracic Organ Transplantation. J Heart Lung Transplant. 2013; 32: 202-211.

https://doi.org/10.1016/j.healun.2012.11.003

[11] Delgado JF, Alonso-Pulpón, Mirabet S, Almenar L, Villas FP, González-Vílchez F, et al. Cancer Incidence in Heart Transplant Recipients With Previous Neoplasia History. Am Jf Transplantation 2016; 16: 1569-1578. https://doi.org/10.1111/ajt.13637

[12] Yoosabai A, Mehta A, Kang W, Chaiwatcharayut W, Sampaio M, Huang M, Nunnapradist S. Pretransplant Malignancy as a Risk Factor for Posttransplant Malignancy After Heart Transplantation. Transplantation 2015; 99: 345350.

https://doi.org/10.1097/TP.0000000000000563

[13] Holdaas H, De Simone P, Zuckermann A. Everolimus and Malignancy after Solid Organ Transplantation: A Clinical Update. J Transplantation 2016

https://doi.org/10.1155/2016/4369574
[14] Kimura Y, Yanase M, Mochizuki H, Iwasaki K, Toda K, Matsuda $S$, et al. De novo malignancy in heart transplant recipients: A single center experience in Japan. J Cardiol 2019; 73: 255-261.

https://doi.org/10.1016/.j.jcc.2018.11.011

[15] Crespo-Leiro MG, Delgado-Jiménez J, López L, AlonsoPulpón L, González-Vilchez F, Almenar-Bonet L, et al. The falling incidence of hematologic cancer after heart transplantation. Clinical Transplantation, 2014, 28: 11421147

https://doi.org/10.1111/ctr.12432

[16] Van Keer J, Droogné W, Van Cleemput J, Vörös G, Rega F, Meyns B, et al. Cancer After Heart Transplantation: A 25year Single-center perspective. Transplantation Proceedings 2016; 48: 2172-2177.

https://doi.org/10.1016/j.transproceed.2016.03.037

[17] Aliabadi A, Grömmer $M$, Cochrane A, Salameh $O$, Zuckermann A. Induction therapy in heart transplantation: where are we now? Transplant International 2013; 26: 684695.

https://doi.org/10.1111/tri.12107

[18] Rudasill SE, lyengar A, Sanaiha $Y$, Khoury H, Mardock AL, Sareh S, Benharash P. Donor history of malignancy: A limited risk for heart transplant recipients. Clinical Transplantation. 2020; 34: e13762.

https://doi.org/10.1111/ctr.13762

[19] Huang S, Tang Y, Zhu Z, Yang J, Zhang Z, Wang L, et al. Outcomes of Organ Transplantation from Donors with a Cancer History. Med Sci Monit 2018; 24: 997-1007. https://doi.org/10.12659/MSM.909059

[20] Mistiaen WP, Van Cauwelaert P, Muylaert P, Wuyts F, Harrisson F, Bortier H. Effect of Prior Malignancy on Survival After Cardiac Surgery. Ann ThoracSurg 2004; 77: 15931597.

https://doi.org/10.1016/j.athoracsur.2003.08.049

[21] Mehra MR, Canter CE, Hannan MM, Semigran MJ, Uber PA Baran DA, et al. The 2016 International Society for Heart LungTransplantation listing criteria for heart transplantation: A 10-year update. J Heart Lung Transplant 2016; 35: 1-23. https://doi.org/10.1016/j.healun.2015.10.023

[22] Saber-Maghaddam N, Nomani H, Sabehkar A, Johnston TP, Mohammadpour $\mathrm{AH}$. The change of immunosuppressive regimen from calcineurin inhibitors to mammalian target of rapamycin (mTOR) inhibitors and its effect on malignancy following heart transplantation. International Immunopharmacology 2019; 9: 150-158. https://doi.org/10.1016/j.intimp.2019.01.035

[23] Gonzalez-Vilchez F, Almenar L, Arizón M, Mirabet S, Delgado B, Diez de Molina B, et al. Temporal Trends in the Use of Proliferation Signal Inhibitors in Maintenance Heart Transplantation: A Spanish Multicenter Study. Transplantation Proc 2010; 42: 2997-3000. https://doi.org/10.1016/j.transproceed.2010.07.060

\section{http://dx.doi.org/10.15379/2410-2822.2020.06.02.01}

(c) 2020 Wilhelm Mistiaen; Licensee Cosmos Scholars Publishing House.

This is an open access article licensed under the terms of the Creative Commons Attribution Non-Commercial License

(http://creativecommons.org/licenses/by-nc/3.0/), which permits unrestricted, non-commercial use, distribution and reproduction in any medium, provided the work is properly cited. 